\title{
Can smart homes extend people with Alzheimer's disease stay at home?
}

\author{
Alberto Brunete González, Micheline Selmes and Jacques Selmes
}

\begin{abstract}
Purpose - The purpose of this paper is to analyse the needs of people with Alzheimer's disease and their caregivers in terms of information and communications technology (ICT) and home automation, and how to foster the use of smart devices in their homes and also, to determine whether the use of ICT can extend people with Alzheimer's disease stay at home in the first stages of the illness, while facilitating their caregivers' tasks.

Design/methodology/approach - Groups of caregivers, ICT researchers and Alzheimer's disease experts were gathered to discuss the utility of several solutions. Sessions were grouped into four topics: safety, leisure, activities of daily living and friendly atmosphere. In total, 23 ICT-based solutions to improve life at home of people with Alzheimer's disease were analysed and grouped under "no interest", "some interest" and "very interesting". Caregivers rated these solutions and suggested improvements to them.

Findings - In total, 18 out of 23 proposals were considered "very interesting", meaning that caregivers considered that they could truly improve the lives of people with Alzheimer's disease. Caregivers also suggested how to progressively introduce these technical solutions into their homes.

Originality/value - ICT and home automation advances could be very useful if used conveniently. Caregivers consider that smart homes can help people with Alzheimer's disease in the security, leisure and daily tasks fields, increasing the time they can live alone in their own homes.
\end{abstract}

\section{Introduction}

About a third of the elders (older than 65 years of age) in Europe live at home alone (Rodrigues et al., 2012) and over the next 20 years, the older population is expected to significantly increase. Amongst elders, those with dementia may need special care to maintain their independent living. People with dementia want and need to live in their own homes, but this is not always a choice. In total, 13 per cent of the world's population aged 60 years and older are dependent (Azheimer's Disease International, 2013). In Europe alone, there are about seven million people with dementia and this number is projected to almost double every 20 years (World Health Organization and Alzheimer's Disease International, 2012). Dementia makes it difficult for people to do many practical things. This will affect their day-to-day life and they may need to adapt how they do things. When supporting the person, there are approaches that carers can take that can reduce the impact of these practical difficulties and help the person maintain a sense of normality for as long as possible. Information and communications technology (ICT) solutions can help carers in many ways.

Alzheimer's disease is the most common form of dementia and possibly contributes to 60-70 per cent of cases (Alzheimer's Association, 2014). This paper will focus on people with Alzheimer's disease and their caregivers. Caring for people with Alzheimer's disease living at home could take many hours a day (up to nine hours in Greece (Rodrigues et al., 2012)). This task is often performed by family members who have to balance their personal and work activities with taking care of their family members. However, caring for people with Alzheimer's disease can be facilitated by new technologies such as home automation and ICT that have emerged in recent years. 
The use of intelligent systems in the homes of people with Alzheimer's disease may help to improve their independence, comfort and safety, avoid depression (Cotten et al., 2014) and facilitate caregivers' daily care tasks. Knowing that the person is at home, that he/she has slept well, that he/she has not had any downtime or safety issues, is an important psychological relief for the caregiver. But, in any case, these technical aids should not be intended to replace caregivers, but rather to facilitate their work. Ignorance of the existing devices and technologies often prevents the use of solutions that could facilitate the care of people with Alzheimer's disease. Home automation technologies have existed for several years and there are already many off-the-shelf commercial devices[1], although not many solutions tailored to people with Alzheimer's and their caregivers. There are already solutions on the market for those who have slight knowledge of electronics and ICT, but few solutions that can be purchased in traditional stores that are easily installable and configurable by people without technological expertise.

With the advent of mobile and new-generation tablets, and especially thanks to the iOS and Android operating systems, a new world of user interfaces has emerged. New services can be accessed without requiring specific knowledge: surfing the Internet, sending emails or executing applications has never been easier (Adlam et al., 2006).

In the last decade, many countries have developed programmes for home care of people with dementia as researched by Nakanishi and Nakashima (2014). These programmes could highly benefit from the use of ICT technologies. For example, in Australia, Transitional Care packages provide short-term therapy and support to elders after a hospital stay to enable them to return home. In (Robert et al., 2016), the importance of ICT for the assessment and rehabilitation in Alzheimer's disease and related disorders is covered. It highlights the use of ICT for caregiver support with people with dementia.

The working group DomAlz (acronym for Domotics and Alzheimer) started out with the aim of bringing together caregivers of Alzheimer and researchers in the field of ICT and home automation, to facilitate access to technology to caregivers and to develop new applications to improve the lives of both people with Alzheimer's disease and caregivers.

This paper presents the solutions and thoughts that have emerged from these discussions between caregivers and researchers in the field of home automation and that are crucial to the development of new smart homes for people with Alzheimer's disease. This work is divided into four main areas that have been chosen as key points: safety, leisure and recreation, activities of daily living (ADLs) and a friendly atmosphere.

\section{Methodology}

A group of five caregivers, three ICT researchers and one Alzheimer expert (from the Spanish Alzheimer Association) was gathered in a room of the Spanish Alzheimer Association to discuss the utility of different ICT solutions, in several sessions, from a qualitative point of view. The group was conducted by one of the ICT researchers.

All caregivers took care of a close relative (parent or spouse/husband) with Alzheimer's disease. The average age of the caregivers was 60 years. The average age of the people they cared for was 70 years. Caregivers had been caring for the person living with dementia for a minimum of five years. Except one, caregivers were not familiar with ICT technology (apart from TV, and first cell phones), but were willing to familiarise themselves with it. Caregivers were in good health (mental and physical) and able to perform daily tasks. They were from Spanish middle-class families.

The ICT experts were researchers of the Universidad Politecnicade Madrid, one with a PhD in Robotics and Automation and two MSc telecommunication engineers.

In a preliminary talk, caregivers were asked about what problems they thought were the most important in their daily life in terms of the care of their relatives in the early stages of the illness. All sessions focused on the early stages of the illness. Then, it was agreed to group the problems into four topics: safety, leisure and recreation, ADLs and a friendly atmosphere. 
A two-hour session was dedicated to each topic. Each session had three parts: introduction, discussion and conclusions. Each of the sessions started with a brief introduction of the topic, presenting currently available solutions in the market to help elders in their daily lives at home. In the discussion phase, caregivers pointed out whether these solutions were useful or not, and in the latter case, how to improve them. At the end of the session, a summary was developed by the main researcher and specific ICT-based solutions were identified and rated by the caregivers under "no interest", "some interest" and "very interesting". The rating was directly provided from the ICT researchers because a qualitative approach to the solution was sought. In the end, topics were divided into 23 ICT-based solutions. As already mentioned, each solution was analysed and grouped under "no interest", "some interest" and "very interesting". Some of these solutions are currently available, but some others will need some time before they can be used in this environment. The estimated adoption time for these solutions was also discussed, considering the experience of the ICT experts and the needs presented by the caregivers.

\section{Results}

The results are presented in Table I. Each row represents topics covered in the previous sections. Each of them is evaluated under "no interest", "some interest" and "very interesting" depending on caregivers' ratings. The estimated adoption time for these solutions is also included.

In total, 18 out of 23 proposals were considered "very interesting", meaning that caregivers considered that they could truly improve the lives of people with Alzheimer's disease, and consequently, extend people with Alzheimer's disease stay at home. Caregivers believed that these technological advances could be very useful if used conveniently. Caregivers also suggested how to progressively introduce these technical solutions into the people's homes. A sudden introduction of many technical solutions could make older people unconfortable.

Table I Study results

No Some High Adoption time (1-now, 2 up to 5 years,
Solution
interest interest interest

\begin{tabular}{|c|c|c|c|}
\hline Fall detection & & $x$ & $1-2$ \\
\hline Safe mobility & & $x$ & 1 \\
\hline Surveillance & & $x$ & 1 \\
\hline Perimeter security & & $x$ & 1 \\
\hline Technical alarms & & $x$ & 1 \\
\hline Adapted interfaces (remote, tablet) & & $x$ & 1 \\
\hline GPS-based apps & & $x$ & 1 \\
\hline Games (tablet, PC) & & $x$ & 1 \\
\hline Multimedia (photos, music, video) & & $x$ & 1 \\
\hline Social networking & & $x$ & 1 \\
\hline Cloth selection & & $x$ & 1 \\
\hline Dressing assistant & & $x$ & 3 \\
\hline Food preparation & & $x$ & $1-2$ \\
\hline Eating habits detection & & $x$ & 1 \\
\hline Eating assistant & $x$ & & 3 \\
\hline Automatic toilet discharging & & $x$ & 1 \\
\hline Electric toothbrush & & $x$ & $1-2$ \\
\hline Liquid dispenser & & $x$ & 1 \\
\hline \multicolumn{4}{|l|}{ Smart Faucet: water temperature } \\
\hline measurement & & $x$ & 1 \\
\hline Smart Faucet: automatic shut down & & $\hat{x}$ & 1 \\
\hline Guiding lights & & $x$ & 1 \\
\hline Sleep monitoring & & $x$ & $1-2$ \\
\hline Automatic shutters & & $x$ & 1 \\
\hline
\end{tabular}




\section{Discussion}

\subsection{Safety}

Most of the caregivers' interest was shown in personal safety, technical alarms and supervision and perimeter security. Personal safety concerns are mainly related to fall detection and safe mobility. Most of the fall detection systems nowadays rely on accelerometers worn by people (bracelets, belts) (Li et al., 2009) or included in smartphones (Aguiar et al., 2014). But they are useless if the person forgets to wear or carry them. Solutions that rely on fixed sensors include cameras and 3D sensors, but most of the solutions based on them are under development (Nguyen et al., 2014; Ozcan et al., 2013).

Many surveillance cameras (IP) nowadays are low cost and have high processing capacity, enabling movement detection and heading direction. Using computer vision algorithms, it is possible to detect falls or longer stays in a certain position, which may be a sign of a problem (i.e. fainting). If motion is not detected in a given period of time, a device (TV, music, etc.) can be activated to make them react. The main problem is that they are expensive systems (since special software is needed) and invade privacy (continuous monitoring).

The use of 3D sensors (like Microsoft's Kinect) to detect persons and movements is exponential (Stone and Skubic, 2015). With this system, given its accuracy and low cost, it is possible to develop fall detection systems incredibly accurately for a reasonable price. The drawback is that it needs to be connected to a PC, which is a major inconvenience if the user wants to place several in a house.

In terms of mobility, it is important that people have good environmental conditions when moving inside the home, for example, detectors to turn lights on or opening the blinds so that the person always has proper lighting. This can be achieved by installing motion detectors and light sensors working in tandem to turn on the lights when a person walks around.

Technical alarms (water and gas leaks, smoke and fire detection) are relatively well covered by commercial devices (and relatively easy to install). A suggestion to prevent fire in kitchen appliances is to use power consumption detectors to find out whether the person switches on the hotplate and forgets to switch it off. These detectors can also be used for the washing machine or the dishwasher to determine how often they are used. This would establish use patterns in the kitchen and eating habits. Combined with intelligent plugs, it is possible to control when appliances are active and when they are not.

Perimeter security is difficult to implement because either keeping the person at home all the time or letting him/her move in and out freely is not completely satisfactory. An intermediary solution is proposed, in which the person has the possibility of leaving home, but warnings are automatically sent to their caregivers. There are many applications that allow notifications when the person leaves a predefined area (may be not only the house, but any area that is established as a park or a shopping area), for example Tweri[2]. It is also very important to ensure that the person has not left the door open when leaving. By automating the door, any of the above systems can also be commanded to close the door.

\subsection{Leisure and recreation}

Smart home devices should also be aimed at entertaining people with Alzheimer's disease and to help them use their free time in the best possible way, either personally performing leisure activities or interacting with their environment, family and other people through the internet.

TV can play an important role as long as it is provided with an adapted interface (Epelde et al., 2011): for example, a remote with few buttons and clearly indicating (large fonts) its function. But it has been found that the use of tablets is a breakthrough because it is simple to use, they are very colourful and bright, there are many different applications (some of them specific to Alzheimer's disease) and they could provide specific interfaces (i.e. launchers in Android). Although people will be using the tablets all day, they should have a pager function to avoid losing them.

When older people are alone, entertainment (photos, music, games, social networking), aids to walk and not getting lost (based on GPS), video conferencing and internet browsing are highlighted applications. Applications that help to maintain brain activity and enhance memory are 
also well considered. For example, animated photo sequences and custom questions recorded by the caregivers help the person to remember places and how to reach them or tasks to be performed and how to perform them. Some other apps allow posting questions (recorded) about specific pictures or parts of them. For example, with the question "where is your daughter?", the person should select the right area of the picture. It is very important for these applications to provide positive feedback. Listening to music from their childhood or adolescence can also be very relaxing.

Carers especially recommended that games should promote social interaction so that the person does not have the feeling of playing alone or against a machine. These games could also be used as assistants to remember many everyday tasks such as meals.

\subsection{Activities of daily living}

One of the main concerns of caregivers was the selection of clothing and to ensure that people with Alzheimer's disease get dressed properly. It may be useful to receive a recommendation on what clothing is appropriate to wear, for example, a smart wardrobe (Goh et al., 2011) to select clothing depending on the weather. A simple solution such as a mobile phone or a microcomputer with internet can download the weather forecast data. If the clothing is classified according to their thermal properties and the device has a database with the cloths of the wardrobe, the device can suggest a combination of clothes on a screen. Also, if the wardrobe is motorised, it can directly bring the desired clothes out.

In terms of eating, carers consider that it is very difficult to provide help to people with Alzheimer's disease. Perhaps a robot could help with this in the future (Takahashi et al., 2001). But considerable help can be provided to the caregivers: keeping track of whether the person has eaten or not (by, e.g. the detection of fridge and microwave use), suggesting ideas for meals, displaying meal-preparation instructions, creating a food inventory or a list of food to be bought. A personal assistant may be essential to help with food storage and the shopping list. But an electronic application can help to maintain a record of the food available in the house.

Solutions that can facilitate grooming may be an automatic toilet water discharging after use, electric toothbrushes with timers, product identification for soap and shampoo or liquid (shower gel) dispensers. As security measures, it is important to mention smart faucets with temperature sensors to avoid scalding with hot water; faucets with automatic opening and closing controlled by presence detection; and indicators of water level (for the bath) and flood detectors.

Caregivers placed considerable emphasis on the system installation to be progressive. In a first step, it should ask the person whether he/she has performed the tasks, "have you combed your hair? Have you washed your face?". In a second phase, it would tell step by step how tasks should be performed. Caregivers do not recommend direct questions (like an exam) but more indirect ones and always with positive comments, "you have combed your hair, haven't you?", or "Surely you've washed your face!".

Reminders that show people with Alzheimer's disease when to do each thing (taking medication, eating, toileting, etc.) are considered very important. These reminders could be displayed through various devices that are in the house, i.e. TV, tablets, cell phones or screens deployed in the house.

\subsection{Friendly atmosphere}

By a friendly atmosphere, it is understood that the person is surrounded by environmental conditions that foster a quiet life. Carers have suggested avoiding disorientation, having good sleep and avoiding fears. It would be very useful to use lights (LEDs) to guide the person at night, especially when the person wakes up at night and wants to go the bathroom or kitchen, which are the most visited rooms at night.

Also, at night, it would be very interesting to use devices that monitor the quality of the sleep and how many times the person gets up to determine how much the person has slept and for how many hours. 
In terms of fears that can appear at home, it would be useful to use stiff curtains (they are not moved by the wind) to avoid strange shadows, and motorised shutters, which can be automatically closed. Automatic window opening and closing could be noiseless and could help ventilate the house when it is quiet outside.

Odour-based systems have been discarded because many people with Alzheimer's disease lose their sense of smell (anosmia). Noise-based systems (such as bells) are also not recommended because noises from unknown sources can frighten people with Alzheimer's disease.

\section{Conclusions}

The importance of home care for people with dementia has been increasing in the last decade, and many countries have developed strategies and policies to reinforce home care services (Nakanishi and Nakashima, 2014). There are many applications where the use of ICT and smart devices in elders' homes can help to extend their independence (Alzheimer's Society, 2016). Many research projects nowadays are adapting the existing technology to the specifications of elderly people and people with degenerative conditions (such as Alzheimer's disease). This article attempts to present new opinions and thoughts about how ICT could help the day-to-day life of people with dementia and their caregivers.

The opinions presented in this paper show that caregivers believe that these technological advances can be very useful if used conveniently. Smart homes could help people with dementia in the security, leisure and daily task fields, increasing the time they can live alone in their own homes. But technical solutions have to be incorporated progressively and at a slow pace in order not to frighten people with dementia.

\section{Notes}

1. www.vesternet.com

2. www.tweri.com/home.aspx 